BUTP-96/12

\title{
New fixed point action for SU(3) lattice gauge theory
}

\author{
Marc Blatter and Ferenc Niedermayer? \\ Institut für theoretische Physik \\ Universität Bern \\ Sidlerstrasse 5, CH-3012 Bern, Switzerland
}

May 1996

\begin{abstract}
We present a new fixed point action for SU(3) lattice gauge theory, which has - compared to earlier published fixed point actions - shorter interaction range and smaller violations of rotational symmetry in the static $q \bar{q}$-potential even at shortest distances.
\end{abstract}

\footnotetext{
${ }^{1}$ Work supported in part by Schweizerischer Nationalfonds

${ }^{2}$ On leave from the Institute of Theoretical Physics, Eötvös University, Budapest
} 


\section{Introduction}

Lattice Monte Carlo simulations provide a powerful tool in studying non-perturbative effects in QCD. Promising applications are, for instance, hadron spectroscopy, high temperature thermodynamics or the structure of the QCD vacuum. However, using a space-time lattice as an ultraviolet regulator introduces discretization errors. A careful analysis is required to extrapolate the Monte Carlo results to zero lattice spacing recovering continuum physics. The simplest choice of the lattice action corresponding to the given continuum theory produces, in general, quite large lattice artifacts. It is advisable to introduce more complicated lattice actions which can significantly reduce such discretization errors.

Wilson's renormalization group (RG) approach [1,2] offers a promising method for eliminating lattice artifacts in asymptotically free field theories [3-8]. Using actions on the renormalized trajectory (RT) of a given RG transformation (RGT) one eliminates lattice artifacts in physical quantities. These were called perfect actions [3]. The RT starts from a fixed point (FP) on the critical surface. The FP action is defined by retaining the functional form of the FP and using it also for finite couplings. In other words, the FP is a point in the space of couplings (including the prefactor $\beta \propto 1 / g^{2}$ ) while the FP action is a straight line going in the direction of $g^{2}$ ). The FP action is a good approximation to the RT. It has the important property that it defines a perfect classical lattice theory: The solutions to the lattice equations of motion have exactly the same value of the lattice action as the continuum action on the corresponding continuum solutions. For example, the FP as a classical action has scale invariant instanton solutions when the corresponding continuum theory has. Furthermore, it can be argued that the RT coincides with the FP action even in 1-loop perturbation theory - the FP action is '1-loop perfect' [2, 6, 9]. In the last years the FP action has been constructed for several two- and four dimensional asymptotically free theories [3 [16].

The type of blocking used in the RGT is to a large extent arbitrary. But the properties of the FP, most notably the interaction range, are very sensitive to the choice of the parameters in the blocking transformation. It has been demonstrated that one can choose the RGT in such a way that the FP action is essentially concentrated on the unit hyper-cube. This is important since one has to simplify the FP action in order to use it in numerical simulations. In measuring physical quantities, like masses, the string tension or critical temperature approximate FP actions seems to give much reduced cut-off effects. But in expectation values of local operators - like the static $q \bar{q}$-potential - new lattice artifacts appear. To reduce such cut-off effects one has to use for a given FP action (or even for an action on the RT) improved local operators. In the RG approach the origin of these artifacts is quite obvious. Although the partition function and all long range properties (masses, etc.) are unchanged by a RG step, the fields on the coarse lattice represent some averages over the original fine fields. If the blocking procedure violates rotational invariance, correlation functions of the coarse fields will show a violation of this symmetry as well. (As an analogy, in continuum electrodynamics the potential between two square-shaped charge distributions is not given by the $1 / r$ potential alone, it has contribution due to higher multipole moments as well. Still the physical content is described exactly, only the testing objects are chosen inconveniently.) One way out, as mentioned above, is to construct better fields (FP fields) another is to use a better, i.e. more rotationally symmetric RGT to make the testing objects, the bare fields, more spherical. The most important requirement remains, of course, the short interaction range of the corresponding FP action.

The present work has been motivated by the fact that the perturbative potential of the bare Polyakov loops for the FP actions considered in ref. [6, 7] has shown a considerable violation of rotational symmetry at a distance of 1-2 lattice units. Our aim is to extend the class of blocking 
transformations to contain 'more rotationally invariant' ones. By optimizing the free parameters of the transformation for a short interaction range, we obtain a FP action which has, as expected, also smaller violation of rotational symmetry in the correlators of bare Polyakov loops. Apart from the complications connected with the more general form of the RGT this work repeats the steps described in detail in ref. [6,77 and we shall try to avoid unnecessary repetitions.

While a parametrization of the FP action should represent the true FP action sufficiently well, it should also be simple enough to be used in numerical simulations. Parametrization errors are one source of 'imperfectness' in the present approach. Another is that the FP action is only an approximation - although a very good one - to the perfect action, i.e. an action on the RT. The latter can be improved by trying to follow the RT [3, 13, 17], the former by including more operators in the parametrization. How far one should go in this direction depends on many things - how large are the remaining lattice artifacts, what is the overhead for including further operators, what is the gain in computer time by working on coarser lattices, etc. A detailed study of the cut-off effects is needed to decide how a given lattice action performs. In this paper only the first few steps are taken in this direction for the new RGT.

The Wilson action in the $\mathrm{SU}(3)$ gauge theory gives huge cut-off effects for thermodynamic quantities (e.g. about $50 \%$ for $N_{t}=4, \beta \rightarrow \infty$ ), therefore it is especially important to use improved actions for such studies [18, 19]. In a related paper A. Papa has studied lattice artifacts in thermodynamics with the new FP action considered here [20].

An alternative way of improving lattice actions is the Symanzik approach 21 24. Here one adds to the action order by order in perturbation theory appropriate irrelevant operators which cancel the leading cut-off effects. Although it is based on perturbation theory, the idea can be extended beyond the perturbative region as well [25]. The tadpole improvement also aims at extending the Symanzik approach beyond the perturbative regime, using a non-perturbative phenomenological determination of the coefficients of the correction terms [26, 27.

We note here that the use of FP actions is especially promising in the study of topological effects [12,14, 16, 28], because in this approach there are no dangerous 'dislocations' and an improved definition of the topological charge can be given.

The paper is organized as follows: First we review the RGT and its FP with special consideration to the new block transformation. We study the quadratic expansion and determine the optimal block transformation parameters. This is followed by the construction of three different loop parametrizations of the FP action. In section 3 we study cut-off effects in the quadratic approximation of the static $q \bar{q}$-potential comparing the Wilson action with the FP actions derived earlier and the new FP action. To set the physical scale we measure the critical couplings for various lattice sizes. This is done in section 3.2. We then study the performance of the new FP action by evaluating the static $q \bar{q}$-potential using Polyakov loop correlators. Finally, in the last section we present some conclusions.

\section{The FP action}

With emphasis on the new block transformation we briefly review the RGT and its FP presented in ref. [6]. We evaluate the FP action in a quadratic approximation and fix the free parameters in the block transformation. Finally, we construct simple parametrizations of the FP action, one of which will be used in MC simulations. 
We consider a $\mathrm{SU}(\mathrm{N})$ pure gauge theory円 in four dimensional Euclidean space defined on a periodic lattice. The partition function is defined as follows

$$
Z=\int D U \mathrm{e}^{-\beta S(U)}
$$

where $D U$ is the invariant group measure and $\beta S(U)$ is some lattice regularization of the continuum action. Starting from such a lattice action we perform a real space RGT by defining a new action by

$$
\mathrm{e}^{-\beta^{\prime} S^{\prime}(V)}=\int D U \exp [-\beta\{S(U)+T(U, V)\}]
$$

Here $T(U, V)$ is the blocking kernel of the transformation and is defined as

$$
T(U, V)=\sum_{n_{B}, \mu}\left(\mathcal{N}_{\mu}\left(n_{B}\right)-\frac{\kappa}{N} \operatorname{Re} \operatorname{Tr}\left[V_{\mu}\left(n_{B}\right) Q_{\mu}^{\dagger}\left(n_{B}\right)\right]\right),
$$

where $Q_{\mu}\left(n_{B}\right)$ is a $N \times N$ matrix which represents some mean of products of link variables $U_{\mu}(n)$ connecting the sites $2 n_{B}$ and $2 n_{B}+2 \hat{\mu}$ on the fine lattice. The term $\mathcal{N}_{\mu}\left(n_{B}\right)$ is a normalization which ensures the invariance of the partition function. The parameter $\kappa$ is a positive constant. It will be fixed later to optimize the quadratic lattice action.

The block transformation is defined by a prescription for constructing the averaging matrices $Q_{\mu}\left(n_{B}\right)$. Two such block transformations were defined in ref. [6]. Here we introduce one, which we call type III blocking. Instead of using just simple staples we also build 'diagonal staples' by going first in the planar or spatial diagonal directions orthogonal to $\hat{\mu}$, then after a step in direction $\hat{\mu}$ returning along the corresponding diagonal. To be specific, first we create the matrices $W^{(m)}\left(n, n^{\prime}\right)$ connecting the sites $n$ and $n^{\prime}$, where $n^{\prime}$ is a site with coordinates $n_{\mu}^{\prime}-n_{\mu}=0,\left|n_{\nu}^{\prime}-n_{\nu}\right| \leq 1$ (for any $\nu$ ):

$$
\begin{aligned}
& W^{(0)}(n, n)=1 \\
& W^{(1)}(n, n+\hat{\nu})=U_{\nu}(n), \\
& W^{(2)}(n, n+\hat{\nu}+\hat{\rho})=\frac{1}{2}\left(U_{\nu}(n) U_{\rho}(n+\hat{\nu})+U_{\rho}(n) U_{\nu}(n+\hat{\rho})\right), \\
& W^{(3)}(n, n+\hat{\nu}+\hat{\rho}+\hat{\lambda})=\frac{1}{6}\left(U_{\nu}(n) U_{\rho}(n+\hat{\nu}) U_{\lambda}(n+\hat{\nu}+\hat{\rho})+\text { permutations }\right) .
\end{aligned}
$$

Here $\nu, \rho$ and $\lambda$ go over all (positive and negative) directions different from $\mu$ and from each other. (Of course, $U_{-\nu}(n)=U_{\nu}^{\dagger}(n-\hat{\nu})$. Values of $W^{(m)}\left(n, n^{\prime}\right)$ not indicated are taken to be zero.) $W^{(2)}\left(n, n^{\prime}\right)$ represents the 'planar diagonal link', $W^{(3)}\left(n, n^{\prime}\right)$ the spatial one. In eqs. 2.4c), 2.4d the sum is taken over all shortest paths leading to the endpoint $n^{\prime}$ of the corresponding diagonal. The fuzzy link operator is constructed then by a modified Swendsen smearing [29]:

$$
\mathrm{W}_{\mu}(n)=\sum_{m=0}^{3} \sum_{n^{\prime}} c_{m} W^{(m)}\left(n, n^{\prime}\right) U_{\mu}\left(n^{\prime}\right) W^{(m)}\left(n^{\prime}+\hat{\mu}, n+\hat{\mu}\right) .
$$

The coefficients $c_{m}$ are free parameters subject to the constraint:

$$
c_{0}+6 c_{1}+12 c_{2}+8 c_{3}=1 .
$$

Finally, the matrix $Q_{\mu}\left(n_{B}\right)$ is the product of two fuzzy link operators connecting the points $2 n_{B}$ and $2 n_{B}+2 \hat{\mu}$ on the fine lattice:

$$
Q_{\mu}\left(n_{B}\right)=\mathrm{W}_{\mu}\left(2 n_{B}\right) \mathrm{W}_{\mu}\left(2 n_{B}+\hat{\mu}\right) .
$$

\footnotetext{
${ }^{1}$ Although the numerical studies have been performed for $\mathrm{SU}(3)$, the equations are mostly given for general $\mathrm{N}$.
} 
The condition eq. (2.6) ensures that for a trivial field configuration $Q_{\mu}\left(n_{B}\right)$ is equal to the unit matrix.

In the limit $\beta \rightarrow \infty$ eq. (2.2) reduces to a saddle point problem. At the FP this is an implicit equation for the FP action $S^{F P}$ :

$$
S^{F P}(V)=\min _{U}\left\{S^{F P}(U)+\sum_{n_{B}, \mu}\left(\mathcal{N}_{\mu}^{\infty}\left(n_{B}\right)-\frac{\kappa}{N} \operatorname{Re} \operatorname{Tr}\left[V_{\mu}\left(n_{B}\right) Q_{\mu}^{\dagger}\left(n_{B}\right)\right]\right)\right\} .
$$

The normalization constant at $\beta=\infty$ becomes

$$
\mathcal{N}_{\mu}^{\infty}\left(n_{B}\right)=\max _{W \in \mathrm{SU}(\mathrm{N})}\left\{\frac{\kappa}{N} \operatorname{Re} \operatorname{Tr}\left[W Q_{\mu}^{\dagger}\left(n_{B}\right)\right]\right\} .
$$

As was already shown in ref. 3, 6 the FP action has scale invariant instanton solutions. This is a consequence of the FP equation (2.8) alone and applies also for type III blocking. We will not proceed along these lines however, but will turn to an expansion in the vector potentials.

\subsection{The quadratic approximation}

Again this section is very similar to the one presented in ref. [6]. The only difference to type I and type II transformations is the actual transformation tensor involved.

The FP equation (2.8) is valid for arbitrary configurations $\{V\}$. For smooth configurations it can be expanded in powers of the vector potentials $B_{\mu}\left(n_{B}\right)$ and $A_{\mu}(n)$ :

$$
V_{\mu}\left(n_{B}\right)=e^{i B_{\mu}\left(n_{B}\right)}, \quad U_{\mu}(n)=e^{i A_{\mu}(n)} .
$$

The most general form of the quadratic action can be written in Fourier space as

$$
2 N S(U)=\frac{1}{V} \sum_{k} \tilde{\rho}_{\mu \nu}(k) \operatorname{Tr}\left[\tilde{A}_{\mu}(-k) \tilde{A}_{\nu}(k)\right]+\mathrm{O}\left(\tilde{A}^{3}\right)
$$

where $V$ is the volume of the fine lattice and $\tilde{\rho}_{\mu \nu}(k)$ are the quadratic coefficients to be determined The transformation kernel becomes

$$
2 N T(U, V)=\frac{\kappa}{V_{B}} \sum_{k_{B}} \operatorname{Tr}\left[\left(\Gamma_{\mu}\left(-k_{B}\right)-B_{\mu}\left(-k_{B}\right)\right)\left(\Gamma_{\mu}\left(k_{B}\right)-B_{\mu}\left(k_{B}\right)\right)\right]+\mathrm{O}(\text { cubic }),
$$

where $V_{B}=V / 16$ is the volume of the coarse lattice. The matrices $\Gamma_{\mu}\left(k_{B}\right)$ represent the linear contributions to the averages $Q_{\mu}\left(n_{B}\right)$ and can be written as

$$
\Gamma_{\mu}\left(k_{B}\right)=\frac{1}{16} \sum_{l=0}^{1} \omega_{\mu \nu}\left(\frac{k_{B}}{2}+\pi l\right) A_{\nu}\left(\frac{k_{B}}{2}+\pi l\right),
$$

where $l=\left(l_{0}, l_{1}, l_{2}, l_{3}\right)$ is an integer vector and the summation goes over $l_{\mu}=0,1$. The tensor $\omega_{\mu \nu}$ is fixed by the form of the blocking kernel:

$$
\omega_{\mu \nu}(k)=\left(1+\mathrm{e}^{i k_{\mu}}\right)\left[c_{0} \delta_{\mu \nu}+6 c_{1} \tau_{\mu \nu}^{(1)}(k)+12 c_{2} \tau_{\mu \nu}^{(2)}(k)+8 c_{3} \tau_{\mu \nu}^{(3)}(k)\right] .
$$

\footnotetext{
${ }^{2}$ In the following we suppress the tilde for Fourier transformed quantities.
} 
Here the coefficients $c_{m}$ were introduced in eq. (2.5). The quantities $\tau_{\mu \nu}^{(m)}(k)$ are the linear contributions to the fuzzy link operator; they can be written as

$$
\begin{aligned}
& \tau_{\mu \nu}^{(1)}(k)=\frac{1}{6}\left[\widehat{k}_{\mu} \widehat{k}_{\nu}^{*}+\delta_{\mu \nu}(6-\xi)\right] \\
& \tau_{\mu \nu}^{(2)}(k)=\frac{1}{24}\left[\widehat{k}_{\mu} \widehat{k}_{\nu}^{*}\left(8-\xi+\widehat{k}_{\mu} \widehat{k}_{\mu}^{*}+\widehat{k}_{\nu} \widehat{k}_{\nu}^{*}\right)+\delta_{\mu \nu}\left(24-8 \xi+\xi{ }^{2}-\eta-\xi \widehat{k}_{\mu} \widehat{k}_{\mu}^{*}\right)\right] \\
& \tau_{\mu \nu}^{(3)}(k)=\frac{1}{48}\left[\widehat { k } _ { \mu } \widehat { k } _ { \nu } ^ { * } \left(24-6 \xi+\xi^{2}-\eta+6 \widehat{k}_{\mu} \widehat{k}_{\mu}^{*}-2 \xi \widehat{k}_{\mu} \widehat{k}_{\mu}^{*}+2\left(\widehat{k}_{\mu} \widehat{k}_{\mu}^{*}\right)^{2}+6 \widehat{k}_{\nu} \widehat{k}_{\nu}^{*}\right.\right. \\
&\left.\quad-2 \xi \widehat{k}_{\nu} \widehat{k}_{\nu}^{*}+2\left(\widehat{k}_{\nu} \widehat{k}_{\nu}^{*}\right)^{2}+2 \widehat{k}_{\mu} \widehat{k}_{\mu}^{*} \widehat{k}_{\nu} \widehat{k}_{\nu}^{*}\right) \\
&+\delta_{\mu \nu}\left(48-24 \xi+6 \xi^{2}-\xi^{3}-6 \xi \widehat{k}_{\mu} \widehat{k}_{\mu}^{*}+2 \xi^{2} \widehat{k}_{\mu} \widehat{k}_{\mu}^{*}-2 \xi\left(\widehat{k}_{\mu} \widehat{k}_{\mu}^{*}\right)^{2}\right. \\
&\left.\left.+3 \xi \eta-2 \epsilon-6 \eta-2 \eta \widehat{k}_{\mu} \widehat{k}_{\mu}^{*}\right)\right] .
\end{aligned}
$$

The lattice momentum $\widehat{k}_{\mu}$ is defined by

$$
\widehat{k}_{\mu}=\mathrm{e}^{i k_{\mu}}-1
$$

and we have introduced the following abbreviations

$$
\xi \doteq|\hat{k}|^{2} \doteq \sum_{\mu} \widehat{k}_{\mu} \widehat{k}_{\mu}^{*}, \quad \eta \doteq \sum_{\mu}\left(\widehat{k}_{\mu} \widehat{k}_{\mu}^{*}\right)^{2}, \quad \epsilon \doteq \sum_{\mu}\left(\widehat{k}_{\mu} \widehat{k}_{\mu}^{*}\right)^{3}
$$

As a consequence of gauge invariance $\tau_{\mu \nu}^{(m)}(k)$ and $\omega_{\mu \nu}(k)$ satisfy the relations

$$
\tau_{\mu \nu}^{(m)}(k) \widehat{k}_{\nu}=\widehat{k}_{\mu}, \quad \omega_{\mu \nu}(k) \widehat{k}_{\nu}=\widehat{2 k}_{\mu} .
$$

Combining the above expansions the FP equation has at the quadratic level the following form

$$
\begin{gathered}
\frac{1}{V_{B}} \sum_{k_{B}} \rho_{\mu \nu}^{\prime}\left(k_{B}\right) \operatorname{Tr}\left[B_{\mu}\left(-k_{B}\right) B_{\nu}\left(k_{B}\right)\right]=\min _{A}\left\{\frac{1}{V} \sum_{k} \rho_{\mu \nu}(k) \operatorname{Tr}\left[A_{\mu}(-k) A_{\nu}(k)\right]\right. \\
\left.+\frac{\kappa}{V_{B}} \sum_{k_{B}} \operatorname{Tr}\left[\left(\Gamma_{\mu}\left(-k_{B}\right)-B_{\mu}\left(-k_{B}\right)\right)\left(\Gamma_{\mu}\left(k_{B}\right)-B_{\mu}\left(k_{B}\right)\right)\right]\right\} .
\end{gathered}
$$

To proceed further we have to introduce a temporary gauge fixing to be able to invert the tensors $\rho_{\mu \nu}$ and $\rho_{\mu \nu}^{\prime}$. One obtains then from eq. (2.19) a recursion relation for the propagator

$$
D_{\mu \nu}(k)=\rho_{\mu \nu}^{-1}(k) .
$$

A way to find the FP solution for the propagator is to start from the Wilson propagator with some gauge fixing parameter $\alpha$ :

$$
D_{\mu \nu}^{(0)}(k)=\frac{\delta_{\mu \nu}}{|\hat{k}|^{2}}+\alpha \frac{\widehat{k}_{\mu} \widehat{k}_{\nu}^{*}}{|\hat{k}|^{4}}
$$

and iterate the propagator to the FP. (At the end we have to switch off the gauge fixing in $\rho_{\mu \nu}$, which means taking the limit $\alpha \rightarrow \infty$.) Using gauge relations for $\omega_{\mu \nu}(k)$ one can show that starting from the standard propagator, after an arbitrary number of iterations, the propagator $D_{\mu \nu}(k)$ assumes the form:

$$
D_{\mu \nu}(k)=G_{\mu \nu}(k)+\alpha f(k) \widehat{k}_{\mu} \widehat{k}_{\nu}^{*},
$$

where $G_{\mu \nu}(k)$ and $f(k)$ are independent of $\alpha$. Under the RGT they are iterated as follows:

$$
G_{\mu \nu}^{\prime}\left(k_{B}\right)=\frac{1}{16} \sum_{l=0}^{1}\left[\omega\left(\frac{k_{B}}{2}+\pi l\right) G\left(\frac{k_{B}}{2}+\pi l\right) \omega^{\dagger}\left(\frac{k_{B}}{2}+\pi l\right)\right]_{\mu \nu}+\frac{1}{\kappa} \delta_{\mu \nu},
$$




\begin{tabular}{|c|c|c|c|}
\hline$r$ & $\rho_{00}(r)$ & $r$ & $\rho_{10}(r)$ \\
\hline $\begin{array}{lllll}0 & 0 & 0 & 1\end{array}$ & -0.47938 & $\begin{array}{llllll}0 & 0 & 0 & 0\end{array}$ & -0.72414 \\
\hline $\begin{array}{lllll}0 & 0 & 1 & 1\end{array}$ & -0.10005 & $\begin{array}{llll}0 & 0 & 0 & 1\end{array}$ & -0.05969 \\
\hline 01111 & -0.03110 & $\begin{array}{lllll}0 & 0 & 1 & 1\end{array}$ & -0.00991 \\
\hline $\begin{array}{llll}1 & 0 & 0 & 1\end{array}$ & 0.00588 & 2100 & -0.00222 \\
\hline 1011 & -0.00457 & $\begin{array}{llll}0 & 1 & 0 & 1\end{array}$ & -0.00204 \\
\hline 1111 & -0.00316 & 2101 & -0.00081 \\
\hline 1002 & 0.00208 & 01111 & -0.00059 \\
\hline 1000 & 0.00204 & 2111 & -0.00043 \\
\hline 2000 & 0.00138 & $\begin{array}{llll}0 & 2 & 0 & 0\end{array}$ & -0.00023 \\
\hline $\begin{array}{llll}0 & 0 & 0 & 2\end{array}$ & 0.00132 & $\begin{array}{llll}0 & 0 & 0 & 2\end{array}$ & 0.00022 \\
\hline $\begin{array}{llll}0 & 0 & 1 & 2\end{array}$ & -0.00084 & $\begin{array}{llll}0 & 0 & 1 & 2\end{array}$ & -0.00011 \\
\hline
\end{tabular}

Table 1: Some of the elements of $\rho_{00}(r)$ and $\rho_{10}(r)$ in configuration space for the RGT of type III. The values are obtained after 5 RG steps on a $16^{4}$ lattice.

and

$$
f^{\prime}\left(k_{B}\right)=\frac{1}{16} \sum_{l=0}^{1} f\left(\frac{k_{B}}{2}+\pi l\right) .
$$

For finite $\alpha$ the action density becomes

$$
\rho_{\mu \nu}(k)=G_{\mu \nu}^{-1}(k)-\alpha f(k) \frac{G_{\mu \rho}^{-1}(k) \widehat{k}_{\rho} \cdot \widehat{k}_{\sigma}^{*} G_{\sigma \nu}^{-1}(k)}{1+\alpha f(k) u(k)},
$$

where we have introduced

$$
u(k)=\widehat{k}_{\sigma}^{*} G_{\sigma \rho}^{-1}(k) \widehat{k}_{\rho} .
$$

In the limit $\alpha \rightarrow \infty$ one obtains

$$
\rho_{\mu \nu}(k)=G_{\mu \nu}^{-1}(k)-\frac{G_{\mu \rho}^{-1}(k) \widehat{k}_{\rho} \cdot \widehat{k}_{\sigma}^{*} G_{\sigma \nu}^{-1}(k)}{u(k)} .
$$

The type III RGT has four real parameters. These are $c_{1}, c_{2}, c_{3}$ and $\kappa$ in eqs. (2.5) and (2.3). We tune them for a short ranged quadratic FP action. Since we optimize in a larger set of blocking transformations (note that at the quadratic level the blocking transformation includes those considered in type I blocking), the final action is expected to have a shorter interaction range. We found the following parameters to be optimal:

$$
c_{1}=0.07, \quad c_{2}=0.016, \quad c_{3}=0.008, \quad \kappa=8.8 .
$$

The iteration procedure converges quite rapidly with a next-to-leading eigenvalue of 0.25 . While for type I and type II blocking the largest couplings of $\rho_{\mu \nu}^{F P}(r)$ decrease as $\exp (-2.7 r)$, rsp. $\exp (-3.1 r)$, for type III blocking it is $\exp (-3.4 r)$. Some of the values of $\rho_{\mu \nu}^{F P}(r)$ have been listed in table 1 .

Solving eq. (2.19) we obtain a linear relation between the field $B_{\mu}$ on the coarse lattice and the minimizing field $A_{\mu}$ on the fine lattice:

$$
A_{\mu}(k)=Z_{\mu \nu}(k) B_{\nu}(2 k),
$$

where

$$
Z_{\mu \nu}(k)=\left[D(k) \omega^{\dagger}(k) D^{\prime-1}(2 k)\right]_{\mu \nu} .
$$




\begin{tabular}{|c|c|c|c|}
\hline$r$ & $Z_{00}(r)$ & $r$ & $Z_{10}(r)$ \\
\hline $\begin{array}{lllll}0 & 0 & 0 & 1\end{array}$ & 0.12507 & 100 & -0.03993 \\
\hline $\begin{array}{lllll}0 & 0 & 1 & 1\end{array}$ & 0.06061 & -1100 & -0.013 \\
\hline $\begin{array}{llll}-1 & 0 & 0 & 0\end{array}$ & 0.05378 & $\begin{array}{llll}-1 & 0 & 0 & 0\end{array}$ & -0.01237 \\
\hline $\begin{array}{llll}-1 & 0 & 0 & 1\end{array}$ & 0.04776 & 110 & -0.0 \\
\hline $\begin{array}{llll}0 & 1 & 1 & 1\end{array}$ & 0.03794 & 200 & 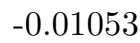 \\
\hline $\begin{array}{llll}-1 & 0 & 1 & 1\end{array}$ & 0.02692 & $\begin{array}{llll}0 & 2 & 0 & 1\end{array}$ & -0 . \\
\hline-20000 & 0.02274 & -1101 & -0.00484 \\
\hline-1111 & 0.01785 & $\begin{array}{lll}0 & 102\end{array}$ & -0.00451 \\
\hline $\begin{array}{llll}0 & 0 & 0 & 2\end{array}$ & 0.01165 & $\begin{array}{llll}0 & 1 & 1 & 1\end{array}$ & -0.00416 \\
\hline $\begin{array}{lllll}-2 & 0 & 0 & 1\end{array}$ & 0.01058 & -1200 & -0.00409 \\
\hline $\begin{array}{llll}-1 & 0 & 0 & 2\end{array}$ & -0.00963 & $\begin{array}{lllll}0 & 0 & 0 & 1\end{array}$ & -0.00406 \\
\hline
\end{tabular}

Table 2: Some of the elements of the tensor $Z_{\mu \nu}(r)$ in configuration space. The lattice size is $16^{4}$ and 5 RG steps were taken.

The connecting tensor $Z_{\mu \nu}$ enters, in particular, in the construction of FP operators [6]. For large $\alpha$ it is given by:

$$
\begin{aligned}
Z_{\mu \nu}(k)= & {\left[G(k) \omega^{\dagger}(k) G^{\prime-1}(2 k)\right]_{\mu \nu}+\frac{1}{u^{\prime}(2 k)} \widehat{k}_{\mu} \cdot{\widehat{2 k_{\sigma}}}_{\sigma}^{*}\left[G^{\prime-1}(2 k)\right]_{\sigma \nu} } \\
& -\frac{1}{u^{\prime}(2 k)}\left[G(k) \omega^{\dagger}(k) G^{\prime-1}(2 k)\right]_{\mu \sigma} \widehat{2 k}_{\sigma} \cdot \widehat{2 k}_{\rho}^{*}\left[G^{\prime-1}(2 k)\right]_{\rho \nu}+\mathrm{O}\left(\frac{1}{\alpha}\right) .
\end{aligned}
$$

where $u^{\prime}(k)$ is defined in eq. (2.26) with $G$ replaced by $G^{\prime}$. The gauge relation for $Z_{\mu \nu}$ reads

$$
Z_{\mu \nu}(k) \widehat{2 k}_{\nu}=\widehat{k}_{\mu} .
$$

Some of the $Z_{\mu \nu}(r)$ values are listed in table 2.

\subsection{Beyond the quadratic approximation}

For the use in numerical simulations we need a parametrization of the FP action $S^{F P}$ in terms of gauge invariant products of link variables $U_{\mu}(n)$. For type I and type II RGT this has been studied in detail in ref. [7]. Here we shall simply outline the procedure and present the results.

We parametrize the FP action by powers of traces of loops:

$$
S(U)=\frac{1}{N} \sum_{\mathcal{C}} \sum_{m} c_{m}(\mathcal{C})\left(\operatorname{Re} \operatorname{Tr}\left[1-U_{\mathcal{C}}\right]\right)^{m},
$$

where $U_{\mathcal{C}}$ denotes the product of link variables $U_{\mu}(n)$ along the closed path $\mathcal{C}$. For a set of quadratically independent loops the coefficients $c_{1}(\mathcal{C})$ can be obtained from the quadratic approximation $\rho_{\mu \nu}(r)$. We apply a numerical procedure to determine those coefficients with $m \geq 2$. We generated about 500 configurations $V$ using the Wilson action with $\beta$ ranging from 5.1 to 50.0 and determined the corresponding fine configurations $U(V)$ by numerical minimization. The procedure is simplified by the observation that the typical value of the action density on the minimizing configuration $U(V)$ in eq. (2.8) is by a factor 30-40 smaller than the action density on the coarse configuration $V$. This allows us two make a two step iteration: First by using a good quadratic approximation on the fine lattice one determines a parametrization of the l.h.s. which includes 
higher powers and describes the 'measured' values of the action well (i.e. those obtained by the minimization procedure). In the next step this precise intermediate parametrization is used on the r.h.s. of eq. (2.8) to determine the value $S^{F P}(V)$ for any configuration $V$, including the very rough ones. The final step is to represent the set $\left\{V^{(i)}, S^{F P}\left(V^{(i)}\right)\right\}$ by some form suitable for numerical simulations. Considering a larger number of loops in eq. (2.33) one gets a better parametrization, but the computer time grows rapidly with the length of the loops, hence one has to restrict the number of loops.

Below we present three alternative parametrizations of the FP action of type III blocking. They are denoted as type IIIa, IIIb, and IIIc. As has been mentioned earlier [3, 6 there are many parametrizations which represent the given set of action values equally well. The optimal values are not unique - the matrix in the corresponding $\chi^{2}$ fit has many nearly zero eigenvalues. Since the roughest configurations dominate the fit our results will represent the FP action on typical configurations generated with $\beta_{\text {Wilson }} \sim 5-6$.

We shall consider the three loops shown in fig. 1. In the parametrizations IIIa and IIIb we

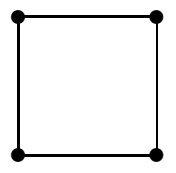

$\mathrm{pl}$

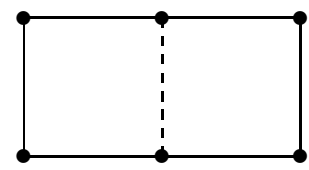

rt

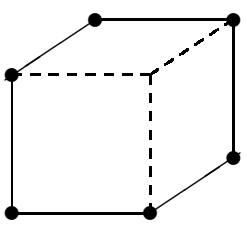

$\mathrm{pg}$

Figure 1: Three different loops considered in the parametrization of the FP action. The plaquette operator ( $\mathrm{pl})$, the rectangle (rt) and the three-dimensional parallelogram (pg).

have chosen the same set of operators as in ref. [7] for the type I FP action, the plaquette and the rectangle. For type IIIc action we have also included the rectangle. The maximal power $m$ in eq. (2.33) for all loops was 4. For the type IIIb and type IIIc parametrization we have used the freedom in the coefficients to demand that they satisfy the tree level Symanzik condition for spectral quantities [23]:

$$
c_{1}(\mathrm{pl})+20 c_{1}(\mathrm{rt})-4 c_{1}(\mathrm{pg})=0 .
$$

The results are listed in table 3. As stated above all the three fits represent the FP values of our

\begin{tabular}{lclccc}
\hline & Operator & $c_{1}$ & $c_{2}$ & $c_{3}$ & $c_{4}$ \\
\hline IIIa & pl & 0.4822 & 0.2288 & -0.1248 & 0.02282 \\
& pg & 0.06473 & -0.02245 & 0.002954 & 0.003471 \\
\hline IIIb & pl & 0.3333 & 0.4845 & -0.2730 & 0.04858 \\
& pg & 0.083333 & -0.01644 & -0.009525 & 0.006324 \\
\hline IIIc & pl & 0.4792 & 0.2226 & -0.1273 & 0.02403 \\
& rt & -0.0091 & -0.04471 & 0.02563 & -0.003698 \\
& pg & 0.0742 & 0.02047 & -0.02398 & 0.007730 \\
\hline
\end{tabular}

Table 3: Couplings of the 8 parameter FP action of type IIIa and type IIIb, and the 12 parameter FP action of type IIIc.

set of configurations equally well. The deviations are similar to those shown in ref. [7]. We expect the three types of parametrizations IIIa, IIIb and IIIc to give comparable results for simulations in the range $\beta_{\text {Wilson }} \sim 5-6$. At small lattice spacings, however, when the linear approximation 
dominates, the Symanzik condition imposed on type IIIb and type IIIc actions will eliminate the $\mathrm{O}\left(a^{2}\right)$ effects, which makes them better suited for perturbative calculations. The couplings in table 3 also satisfy the normalization condition

$$
c_{1}(\mathrm{pl})+8 c_{1}(\mathrm{rt})+8 c_{1}(\mathrm{pg})=1 .
$$

Note that the whole quadratic part in the full FP action satisfies exactly the Symanzik condition. However, in a parametrization using a restricted set of loops this condition may not be satisfied any more - unless it is specially requested as an auxiliary condition. The quadratic part (the coefficients $c_{1}(\mathcal{C})$ ) used in the parametrization IIIc are obtained from the best fit to the quadratic coefficients $\rho_{\mu \nu}(r)$ - with those operators under the constraint (2.34). All the numerical simulations have been performed using the parametrization IIIa. The other two forms are given only to show the ambiguity of the truncation step and to provide a parametrization which maintains the Symanzik improved nature of the full FP action.

\section{$3 \quad$ The static $q \bar{q}$-potential}

A good check for discretization errors is offered by the static $q \bar{q}$-potential. Below the critical temperature $T_{c}$ the potential is expected to rise linearly for larger distances, with the string tension as its slope. For smaller distances the potential should exhibit a Coulomb potential. In lowest order perturbation theory it is proportional to the time average of the zero component of the free field propagator. In the following two sections we study the perturbative potential to lowest order and the full potential using Polyakov loop operators.

\subsection{The tree level potential}

At the quadratic level the static potential in a finite continuum box 1 of size $L_{s}^{3}$ is proportional to the Coulomb potential:

$$
V_{\text {cont }}(\vec{x})=\frac{1}{L_{s}^{3}} \sum_{\vec{k} \neq 0} \mathrm{e}^{i \vec{k} \vec{x}} \frac{1}{(\vec{k})^{2}}, \quad \text { where } \quad k_{i}=\frac{2 \pi}{L_{s}} l_{i} .
$$

On a lattice of size $N_{s}^{3}$ and an arbitrary lattice action we get

$$
V(\vec{r})=\frac{1}{N_{s}^{3}} \sum_{\vec{k} \neq 0} \mathrm{e}^{i \vec{k} \vec{r}} D_{00}\left(k_{0}=0, \vec{k}\right)
$$

where $D_{\mu \nu}(k)$ is the propagator corresponding to the quadratic part of the lattice action. On fig. 2 we plotted the differences between the lattice potentials and the continuum potential for Wilson action and type I, II, and III actions. To see how large this deviations are one can compare the force measured on the lattice to the continuum force. In table 1 the relative differences

$$
R\left(\vec{r}_{1}, \vec{r}_{2}\right)=\frac{\left(V\left(\vec{r}_{1}\right)-V_{\text {cont }}\left(\vec{r}_{1}\right)\right)-\left(V\left(\vec{r}_{2}\right)-V_{\text {cont }}\left(\vec{r}_{2}\right)\right)}{V_{\text {cont }}\left(\vec{r}_{1}\right)-V_{\text {cont }}\left(\vec{r}_{2}\right)}
$$

are listed for the few first distances on the lattice. There are two types of deviations from the

\footnotetext{
${ }^{1}$ Note that the force at $|\vec{x}| / L \approx 1 / 3$ in a finite box differs from the infinite volume result by as much as $25 \%$ so it is essential to use the correct finite box expression for comparison to the lattice results.
} 


\begin{tabular}{ccrrrr}
\hline$\vec{r}_{1}$ & $\vec{r}_{2}$ & Wilson & \multicolumn{1}{c}{ type I } & type II & type III \\
\hline$(0,1,1)$ & $(0,0,1)$ & 0.325 & 0.2797 & 0.1479 & -0.0080 \\
$(1,1,1)$ & $(0,1,1)$ & 0.125 & 0.0051 & 0.0113 & -0.0044 \\
$(0,0,2)$ & $(1,1,1)$ & -0.895 & -0.0544 & -0.0725 & 0.0125 \\
$(0,1,2)$ & $(0,0,2)$ & 0.662 & 0.0034 & 0.0493 & -0.0048 \\
$(1,1,2)$ & $(0,1,2)$ & 0.283 & 0.0092 & 0.0118 & -0.0022 \\
$(0,2,2)$ & $(1,1,2)$ & -0.103 & -0.0016 & -0.0022 & 0.0004 \\
$(1,2,2)$ & $(0,2,2)$ & 0.183 & 0.0017 & 0.0023 & -0.0005 \\
\hline
\end{tabular}

Table 4: The relative error of the perturbative force on the lattice (cf. eq. (3.3)) for various lattice actions.

continuum result for the FP action [6]. The first type is due to the deviation of the shape of the blocking from a spherical averaging. This part contains, for example, a term $\propto P_{4}(\cos \theta) / r^{5}$ (an octupole term) and can be corrected by using FP Polyakov loops $\$$. The second type is an exponentially falling correction $\propto \exp \left(-r / r_{0}\right)$ where $r_{0}$ is of the order of the interaction range and is due to the effect of the quantum fluctuations on the fine lattice. As seen from fig. 2, for type I and type II actions the discretization errors are much reduced, but at $r=1$ a substantial deviation from the continuum can be observed. On the other hand, for type III action the perturbative potential lies for all distances very near to the continuum result.

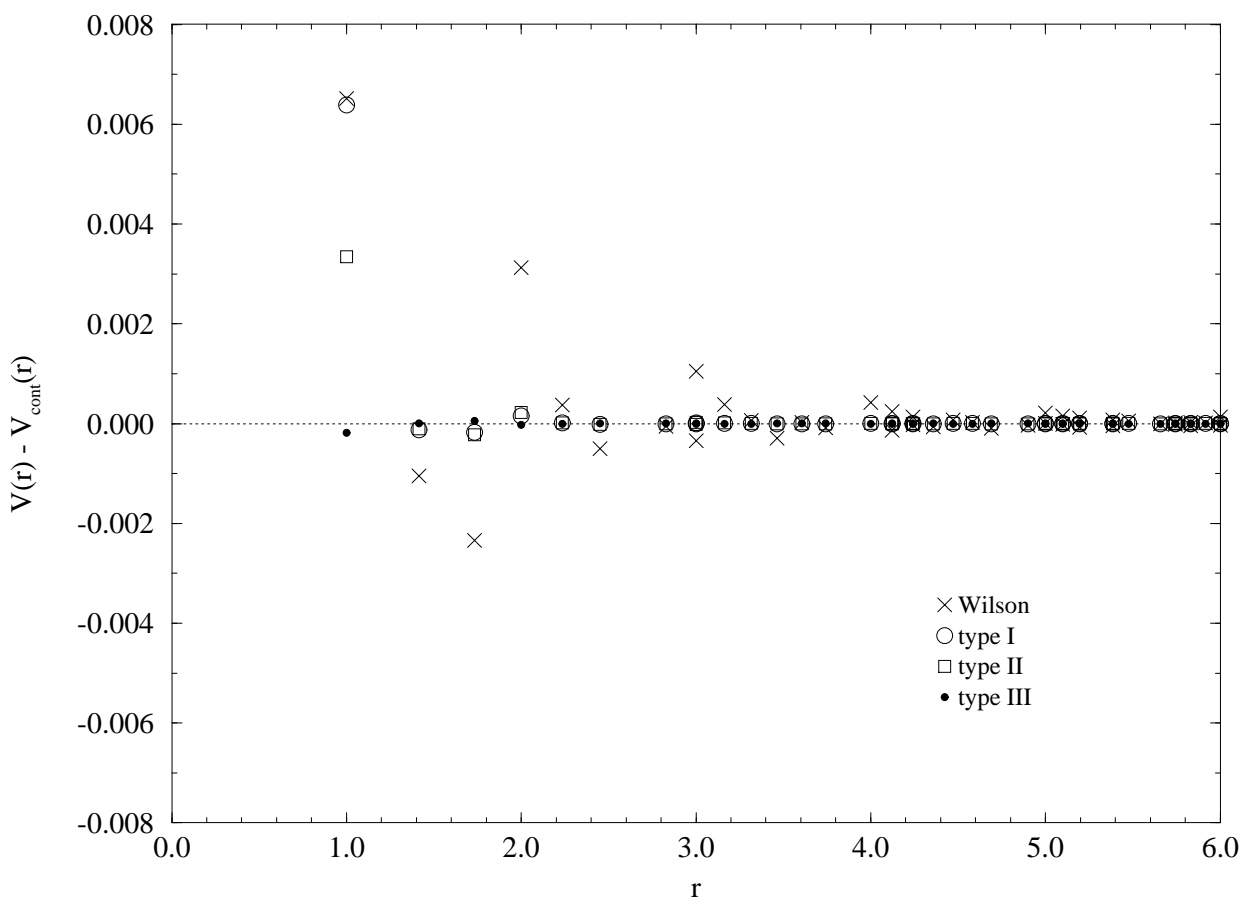

Figure 2: A comparison of the quadratic potential for different blocking prescriptions in a cubic box of size $16^{3}$. A non-physical constant was subtracted to match the curves.

\footnotetext{
${ }^{2}$ We have checked this explicitly by constructing FP Polyakov loops in the linear approximation.
} 


\subsection{The temperature scale}

In this section we determine the critical couplings $\beta_{c}$ for the colour deconfinement phase transition on lattices with different temporal extensions $N_{t}$. As in ref. [7] we use the definition of the Columbia group [30]: For a given lattice size $N_{s}^{3} \times N_{t}$ we perform MC simulations for various couplings $\beta$ around the expected transition region measuring the Polyakov loop averaged over the lattice:

$$
P=\frac{1}{N_{s}^{3}} \sum_{\vec{n}} \operatorname{Tr} \prod_{n_{0}=0}^{N_{t}-1} U_{0}\left(n_{0}, \vec{n}\right)
$$

We evaluate the angle $\theta$ from $\tan (\theta)=\operatorname{Im}(P) / \operatorname{Re}(P)$ and measure the fraction of the time $f_{20}$ that $\theta$ lies in the range of $\pm 20^{\circ}$ of the directions of the $\mathrm{Z}(3)$ roots. Finally, the phase transition is defined as the coupling $\beta$ where the simulations yield for the deconfinement fraction

$$
f_{d}=\frac{3}{2} f_{20}-\frac{1}{2}
$$

the value 0.5 .

We measured the critical couplings for lattices of temporal extensions $N_{t}=2,3,4$ and 6 with different spatial sizes $N_{s}$. The results are listed in table 5. The deconfinement fraction has been determined at various couplings in the vicinity of the phase transition. At each coupling we have performed from 2000 to 30000 sweeps. The critical coupling $\beta_{c}$ was obtained by a linear fit of the fractions bracketing $f_{d}\left(\beta_{c}\right)=0.5$. Its error was obtained by a jack-knife analysis. The

\begin{tabular}{ccccc}
\hline volume & $N_{t}=2$ & $N_{t}=3$ & $N_{t}=4$ & $N_{t}=6$ \\
\hline $4^{3}$ & $3.361(5)$ & & & \\
$5^{3}$ & $3.378(3)$ & $3.568(4)$ & & \\
$6^{3}$ & $3.385(9)$ & & $3.678(3)$ & \\
$8^{3}$ & $3.395(3)$ & $3.581(4)$ & & \\
$9^{3}$ & $3.399(5)$ & & $3.686(3)$ & $3.91(4)$ \\
$10^{3}$ & & $3.587(5)$ & $3.691(5)$ & $3.87(1)$ \\
$12^{3}$ & & & $3.691(5)$ & $3.882(7)$ \\
$14^{3}$ & & & & $3.882(8)$ \\
$16^{3}$ & $3.400(3)$ & $3.588(4)$ & $3.695(4)$ & $3.886(13)$ \\
$\infty$ & & & \\
\hline
\end{tabular}

Table 5: Critical couplings at finite volume and extrapolated to infinite volume for the FP action with parameters IIIa in Table 3 .

critical couplings in the thermodynamic limit $\left(N_{s} \rightarrow \infty\right)$ were obtained by extrapolating the finite couplings using the finite size law 19,30 :

$$
\beta_{c}\left(N_{t}, N_{s}\right)=\beta_{c}\left(N_{t}, \infty\right)-c\left(N_{t}\right)\left(\frac{N_{t}}{N_{s}}\right)^{3} .
$$

The finite size scaling behaviour is plotted in fig. 3 .

\subsection{The full potential}

To repeat similar measurements of ref. [7] we have measured the static $q \bar{q}$-potential $V(r ; T)$ at finite temperature $T=\frac{2}{3} T_{c}$ for the type III action. The result for $\beta=\beta_{c}\left(N_{t}=2\right)=3.40$ on a lattice 


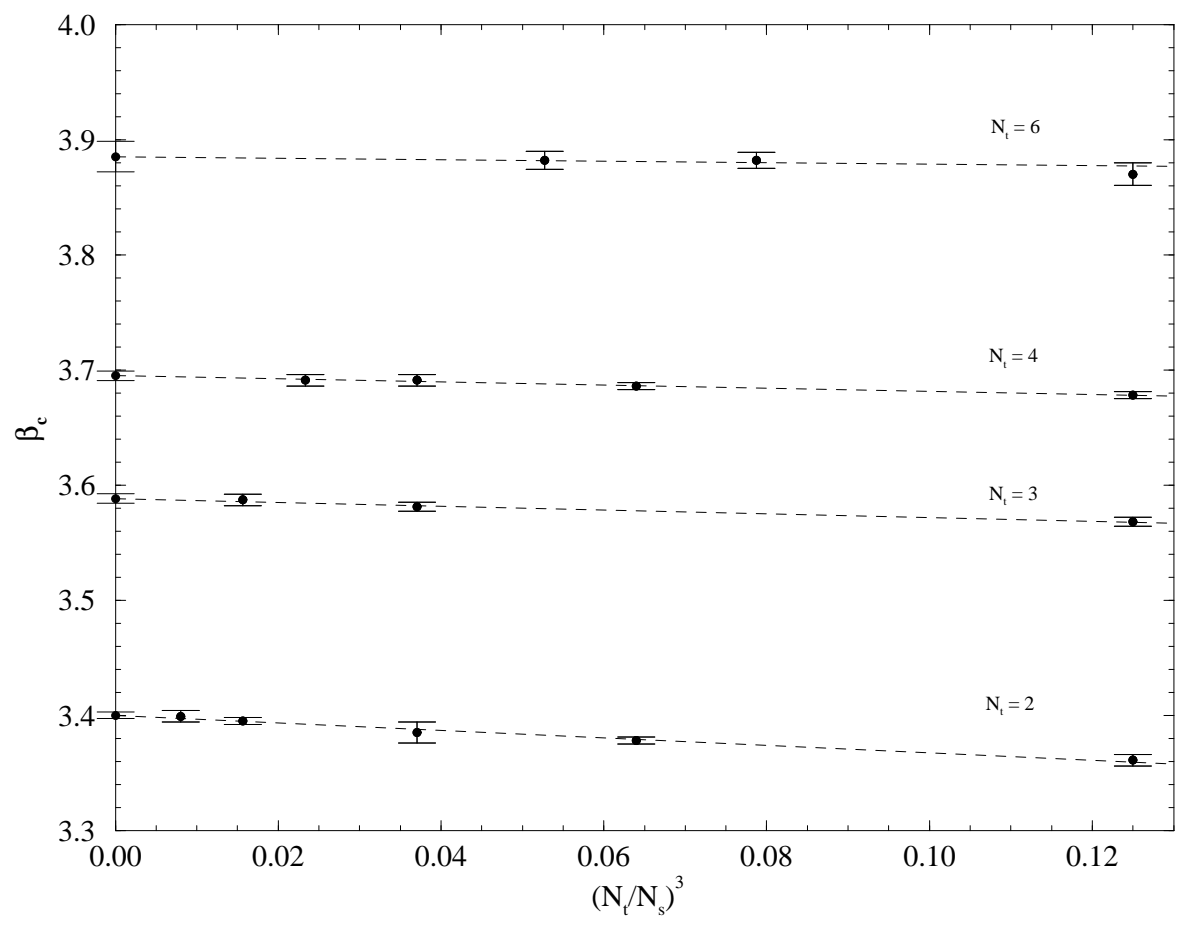

Figure 3: Finite size scaling for the critical couplings of the deconfinement phase transition using the parametrization IIIa. The $\beta=\infty$ extrapolations are included.

$6^{3} \times 3$ is shown on fig. 4 . The constant in the potential is fixed by setting $V\left(r=1 / T_{c} ; T\right)=0$. For the Wilson action we used $12^{3} \times 6$ and $6^{3} \times 3$ lattices, again at $T=\frac{2}{3} T_{c}$. These data and those for type I action are taken from ref. [7]. As opposed to the Wilson action, both FP actions for $N_{t}=3$ have a proper slope at large distances. One also observes that the type III action has a somewhat smoother potential than type I. The difference is, however, not as striking as for the perturbative potential on fig. 2. Note also a phenomenon which looks strange at first sight. The third point from the right on the type I and type III plots - which corresponds to the diagonal distance $(2,2,2)$ - has larger error bars than the others. In the Wilson case this point is not plotted because the measured correlation value was lost in the noise. This is also a sign of strong violation of rotational symmetry, and is not completely restored in the parametrized FP actions either. The reason for this phenomenon (besides the trivial factor of different multiplicities) is, perhaps, that in the Wilson action there is no direct interaction between diagonally separated links, and in the actual parametrization of the FP actions used here no spatial diagonal interaction term is included - this would be an 8-link 'parallelogram'. (Such direct interaction term is certainly present in the true FP action.) As a result, the diagonally separated Polyakov loops are not bound to each other so strongly as they should be. A similar behaviour is observed for the distance $(1,1,1)$, but the errors are too small there to be seen in the figure. 

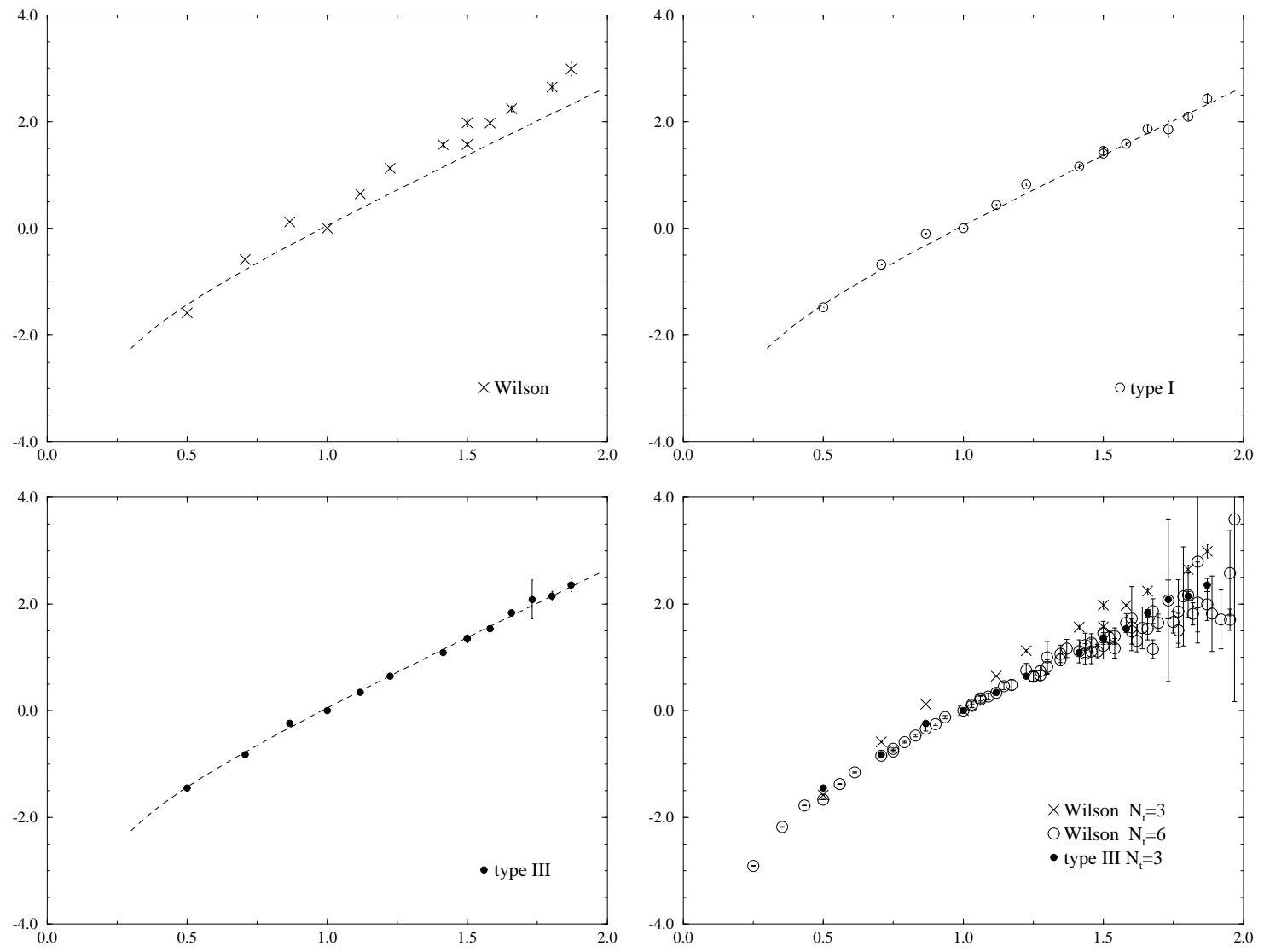

Figure 4: The $q \bar{q}$-potential $V(r ; T) / T_{c}$ vs. $r T_{c}$ for different actions at $T=2 / 3 T_{c}$. Upper left: Wilson, upper right: type I, lower left: type III action. These three plots correspond to $N_{t}=3$. The dashed line shows the function $-0.24 / r+2.48 r-2.19$, and serves to guide the eye. The lower right graph shows again the potential for type III and Wilson action for $N_{t}=3$, together with the $N_{t}=6$ result for the Wilson action. The spatial size in all cases is $N_{s}=2 N_{t}$. 


\section{Conclusion}

Using a more general smearing kernel we obtained a new FP action which in the quadratic approximation performs better than those obtained previously - it has a somewhat faster decay rate for the interaction coefficients and produces a perturbative potential close to the continuum one. As far as the cut-off effects for finite $\beta$ are concerned, the performance of this new FP action, or rather of the actual parametrization suggested here, has to be investigated further. To improve the parametrization it could also be useful to include rough classical solutions (like instantons or constant Abelian fields) with analytically known action values [12,14].

As mentioned in the introduction, A. Papa has measured the cut-off effects in the free energy for type I and type IIIa actions [20]. For the type IIIa action at $T=2 T_{c}$ on a lattice with temporal extension $N_{t}=3$ no cut-off effect has been observed within the small errors - the result agrees with the continuum prediction of ref. [18, 19] and even at $N_{t}=2$ the error is $\sim 10 \%$. For the type I the deviation is $\sim 10 \%$ at $N_{t}=3$. This has to be compared to the Wilson case where the cut-off effect is $\sim 100 \%$ at $N_{t}=2$ and even at $N_{t}=4$ it is still $\sim 20 \%$. Because the necessary simulation

time grows very fast with $N_{t}$ - about $N_{t}^{10}$ - it is essential to keep the cut-off effects as small as possible.

\section{Acknowledgments:}

We wish to thank Ruedi Burkhalter, Tom DeGrand, Anna Hasenfratz and Alessandro Papa for useful discussions. Especially, we would like to thank Peter Hasenfratz for many helpful conversations and for reading the manuscript. The codes not specific to type III RGT were taken over from previous work [6, ].

\section{References}

[1] K. Wilson and J. Kogut, Phys. Rep. C12 (1974) 75; K. Wilson, Rev. Mod. Phys. 47 (1975) 773, ibid., 55 (1983) 583.

[2] K. Wilson, in Recent developments in gauge theories, ed. G.'t Hooft et al. (Plenum, New York, 1980).

[3] P. Hasenfratz and F. Niedermayer, Nucl. Phys. B414 (1994) 785.

[4] P. Hasenfratz, Nucl. Phys. B (Proc. Suppl) 34 (1994) 3; F. Niedermayer, ibid., 513.

[5] T. DeGrand, A. Hasenfratz, P. Hasenfratz, F. Niedermayer and U.-J. Wiese, Nucl. Phys. B (Proc. Suppl.) 42 (1995) 67.

[6] T. DeGrand, A. Hasenfratz, P. Hasenfratz, F. Niedermayer, Nucl. Phys. B454 (1995) 587.

[7] T. DeGrand, A. Hasenfratz, P. Hasenfratz, F. Niedermayer, Nucl. Phys. B454 (1995) 615.

[8] T. DeGrand, A. Hasenfratz, P. Hasenfratz and F. Niedermayer, Phys. Lett. B 365 (1996).

[9] F. Farchioni, P. Hasenfratz, F. Niedermayer and A. Papa, Nucl. Phys. B 454 (1995) 638.

[10] U.-J. Wiese, Phys. Lett. B315 (1993) 417;

W. Bietenholz and U.-J. Wiese, MIT preprint, CTP 2423 (1995), hep-lat/9503022;

W. Bietenholz, U.-J. Wiese, MIT preprint CTP-2475, hep-lat/9510026. 
[11] W. Bietenholz, E. Focht and U.-J. Wiese, Nucl. Phys. B436 (1995) 385. Nucl. Phys. B (Proc. Suppl.) 42 (1995) 790.

[12] M. Blatter, R. Burkhalter, P. Hasenfratz and F. Niedermayer, Nucl. Phys. B (Proc. Suppl.) 42 (1995) 799;

M. Blatter, R. Burkhalter, P. Hasenfratz and F. Niedermayer, Phys. Rev. D 53 (1995) 923.

[13] W. Bock and J. Kuti, Phys. Lett. B 367(1996)242.

[14] R. Burkhalter, preprint BUTP-95/18, hep-lat/9512032.

[15] U. Kerres, G. Mack, and G. Palma, preprint DESY 94-226 (1995).

[16] T. DeGrand, A. Hasenfratz and D. Zhu, COLO-HEP-369, hep-lat/9603015;

T. DeGrand, A. Hasenfratz and D. Zhu, COLO-HEP-370, hep-lat/9604009.

[17] A.P. Gottlob, M. Hasenbusch and K. Pinn, MS-TPI-96-1, hep-lat/9601014.

[18] B. Beinlich, F. Karsch and E. Laermann BI-TP 95/33, CERN-TH/95-253, hep-lat/9510031.

[19] G. Boyd, J. Engels, F. Karsch, E. Laermann, C. Legeland, M. Lütgemeier and B. Petersson, preprint BI-TP 96/04, hep-lat/9602007.

[20] A. Papa, preprint BUTP-96/13, hep-lat/9605004.

[21] K. Symanzik, in "Recent Developments in Gauge Theories," eds. G. 't Hooft, et. al. (Plenum, New York, 1980) 313;

K. Symanzik in "Mathematical Problems in Theoretical Physics", eds. R. Schrader et. al. (Springer, New York, 1982);

K. Symanzik Nucl. Phys. B226 (1983) 187, 205.

[22] M. Lüscher, K. Symanzik and P. Weisz, Nucl. Phys. B173 (1980) 365,

Ph. de Forcrand, G. Schierholz, H. Schneider and M. Tepper, Phys. Lett. B160 (1985) 137.

[23] P. Weisz Nucl. Phys. B212 (1983) 1;

M. Lüscher and P. Weisz, Nucl. Phys. B240[FS12] (1984) 349;

G. Parisi, Nucl. Phys. B254 (1985) 58;

M. Lüscher and P. Weisz, Comm. Math. Phys. 97 (1985) 59.

[24] M. Lüscher and P. Weisz, Comm. Math. Phys. 97 (1985) 59, 98 (1985) 433;

M. Lüscher, In Les Houches 1984, 359-374.

[25] K. Jansen, Ch. Liu, M. Lüscher, H. Simma, S. Sint, R. Sommer, P. Weisz and U. Wolff, preprint DESY 95-230, hep-lat/9512009.

[26] G.P. Lepage and P. Mackenzie, Phys. Rev. D48 (1993) 2250.

[27] M. Alford, W. Dimm, G.P. Lepage, G. Hockney and P.B. Mackenzie, Phys. Lett. B 361 (1995) 87 ;

M. Alford, W. Dimm, G.P. Lepage, G. Hockney, P.B. Mackenzie, Nucl. Phys. B, Proc. Suppl. 42 (1995) 787;

G. P. Lepage, talk at Lattice 95, Melbourne, Australia, hep-lat/9510049.

[28] M. D'Elia, F. Farchioni and A. Papa, preprint IFUP-TH-60-95, hep-lat/9511021.

[29] R. H. Swendsen, Phys. Rev. Lett. 52 (1984) 2321;

A. Hasenfratz, P. Hasenfratz, U. Heller, and F. Karsch, Phys. Lett. 140B (1984), 76;

K. C. Bowler, et. al., Nucl. Phys. B257[FS14] (1985), 155 and Phys. Lett. 179B (1986) 375;

QCD-TARO collaboration (K. Akemi, et. al.), Phys. Rev. Letters 71 (1993), 3063.

[30] N. H. Christ and A. E. Terrano, Phys. Rev. Lett. 71 (1986) 11. 